\title{
Improved Model of Photovoltaic Systems
}

\author{
Dmitry Altshuller ${ }^{1}$ Peter Hüsson ${ }^{1}$ Christopher Alain Jones ${ }^{1}$ Leonard Janczyk ${ }^{2}$ \\ ${ }^{1}$ Dassault Systemes, USA, \{dmitry.altshuller, peter.huesson, \\ christopher.jones\} \& $3 \mathrm{ds}$. com \\ ${ }^{2}$ Dassault Systemes, Germany, leonard.janczyk@3ds.com
}

\begin{abstract}
The paper describes a model of a typical photovoltaic (PV) system. Unlike models previously discussed in literature, heat transfer phenomena are accounted for simultaneously with the electrical dynamics. Furthermore, the model is simulated for a time scale of one full year.
\end{abstract}

Keywords: Photovoltaic system, Modelica, Thermal effect, Modeling thermal effect, Solar power, Dymola

\section{Introduction}

The importance of obtaining energy from renewable resources cannot be overestimated. However, harnessing these resources often presents considerable technical difficulties. Furthermore, the effectiveness of using resources such as wind or solar power depends on the weather conditions. It is, therefore, critically important to develop mathematical models that can reliably predict power output before any significant investment is made.

The main difficulty in modeling photovoltaic (PV) systems lies in the complexity of accounting for all the factors that may influence the performance of a PV cell. Most of the existing models of PV systems tend to focus only on some of these factors while simplifying the influence of others. For example, modeling the influence of solar irradiance is emphasized in (Tian et al, 2012) as well as in (Khatib and Elmenreich, 2016) while the dependence of temperature is simplified. By contrast, a detailed thermal model is developed in (Jones and Underwood, 2001) but the influence of electric power output on the temperature of the system, which, in turn, affects this power output, is considerably simplified.

The most commonly used tool for modeling PV systems is Simulink. PSpice is used in (Castaner and Silvestre, 2002) and MATLAB is used in (Khatib and Elmenreich, 2016). In this paper we propose to use Modelica and the tool Dymola to account for the mutual influence of the power output and temperature variation. To this end, we start with the circuit model proposed in (Pandiarajan and Muthu, 2011) which is then combined with the thermal model from (Jones and Underwood, 2001). The simulation is run using the weather module from the HVAC Library developed by XRG Simulation.

\section{Mathematical Background}

\subsection{Equivalent Circuit}

The concept of using an equivalent circuit to model a PV cell goes back to the book (Angrist, 1982). The model was subsequently improved in the book (Masters, 2004) and is to this day used. The circuit consists of a signal-dependent current source connected anti-parallel to a diode, parallel to a resistor, and in series with another resistor. The generic schematic of the equivalent circuit is shown in Figure 1.

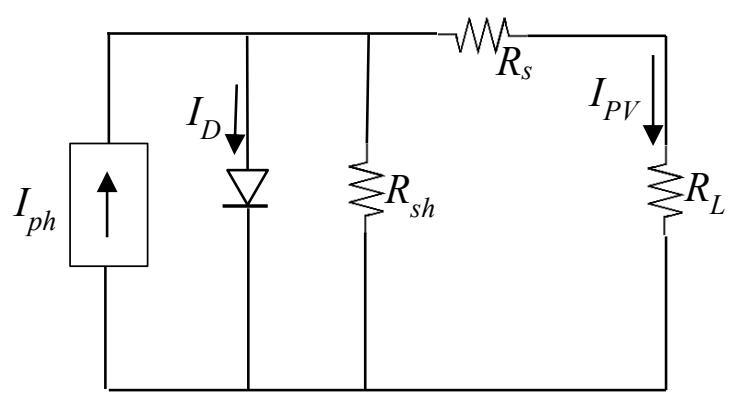

Figure 1. Schematic of a PV equivalent circuit.

The resistors $R_{s h}$ and $R_{s}$ are the intrinsic shunt and series resistances of the cell. Typically, the value of $R_{s h}$ is several magnitues higher than $R_{s}$. Therefore, they can be neglected to simplify some of the equations. These parameters also affect the characteristic of the diode.

The current $I_{p h}$ depends on the solar radiation and the temperature of the system. In addition, the diode current depends on several other voltages and currents in the circuit. In the next subsections we will give the details of these models.

\subsection{Modeling of the Current Source}

The current source is the element where the solar irradiance energy is converted into the electrical energy. The equation for the current $I_{p h}$ has the form (Seyedmahmoudian et al, 2013):

$$
I_{p h}=\left[I_{S C}+K_{i}\left(T-T_{r e f}\right)\right] \frac{G}{G_{r e f}}
$$

In this equation $I_{S C}$ is the short-circuit current, $K_{i}$ is the temperature coefficient, $T$ is the temperature of the 
$\mathrm{PV}$ cell, $T_{r e f}=298 \mathrm{~K}$ is the reference temperature, $G$ is the solar irradiance, and $G_{r e f}=1000 \mathrm{~W} / \mathrm{m}^{2}$ is its reference value.

The value of the solar irradiance is obtained from the weather model which is taken from the HVAC Library developed by XRG Simulation. The temperature is obtained by modeling various heat transfer phenomena described in Subsection 2.4.

\subsection{Modeling of the Diode}

The diode current is described by the following equation (Pandiarajan and Muthu, 2011):

$$
I_{D}=I_{0}\left[\exp \frac{q\left(V_{P V}+I_{P V} R_{S}\right)}{N_{S} A k T}-1\right]
$$

The parameters in this equation are as follows: $q=1.6 \mathrm{E}-19 \mathrm{C}$ is the electron charge;

$N_{S}$ is the number of PV cells connected in series;

$A$ is the ideality factor;

$k=1.3805 \mathrm{E}-23 \mathrm{~J} / \mathrm{K}$ is the Boltzmann constant.

Also, involved in the equation are voltage through and current at the load. These are obtained while the simulation is running.

The saturation current $I_{0}$ is calculated from (Angrist, 1982):

$$
I_{0}=I_{R S}\left(\frac{T}{T_{\text {ref }}}\right)^{3} \exp \frac{q E_{\mathrm{g} 0}\left(T-T_{\text {ref }}\right)}{B k T T_{\text {ref }}}
$$

In addition to the already defined variables and parameters, $B$ is the ideality factor and $E_{\mathrm{g} 0}$ is the band gap. The equation for the reverse saturation current $I_{R S}$ is (Pandiarajan and Muthu, 2011):

$$
I_{R S}=\frac{I_{S C}}{\exp \frac{q V_{O C}}{N_{S} k A T}-1}
$$

The new parameters in this equation are the short-circuit current $I_{S C}$ and the open-circuit voltage $V_{O C}$.

The reader is referred to the paper (Pandiarajan and Muthu, 2011) and references therein for a more detailed discussion of these equations and parameters.

\subsection{Modeling of the Heat Transfer}

There are four heat transfer mechanisms that must be modelled: heating by short-wave and long-wave radiation as well as cooling by free and forced convection. In addition, there is heat loss equal to the power output of the PV system. Let us describe each of these following (Jones and Underwood, 2001).

The short-wave radiation is directly proportional to the solar irradiance and is equal to $\alpha A G$, where $\alpha$ is the absorptivity, $A$ is the surface area, and $G$ is solar irradiance.
The long-wave radiation comes from two sources: sky and ground. It obeys the Stefan-Boltzmann law but the terms must be multiplied by respective emissivity of the sky, the ground, and the PV module. Furthermore, for the purposes of radiation modeling, the temperature of the sky is increased by $20 \mathrm{~K}$ for clear sky. This number is reduced down to zero for the overcast sky, proportionately to cloud cover. In addition, adjustments need to be made for the tilt angle of the panel.

The convection flow rate is directly proportional to the difference between the temperature of the panel and the ambient temperature. The coefficient for the free convection is proportional to the cubic root of this temperature difference (Holman and Bhattacharyya, 2011, p.335) but the coefficient for the forced convection is treated as a parameter since there are presently no known reliable correlations for it.

The equation has the form (Jones and Underwood, 2001):

$$
\begin{aligned}
C \frac{d T}{d t}=\alpha A G+ & \sigma A \\
& +\frac{1-\cos \beta}{2} \varepsilon_{\text {ground }} T_{\text {ground }}^{4} \\
& \left.-\varepsilon_{\text {module }} T_{\text {module }}^{4}\right) \\
& -\left(h_{c, \text { forced }}\right. \\
& \left.+1.31 \sqrt[3]{T-T_{\text {amb }}}\right) A(T \\
& \left.-T_{\text {amb }}\right)-P_{\text {out }}
\end{aligned}
$$

In this equation, in addition to the already defined variables and parameters, $C$ is the heat capacity of the module, $\sigma$ is the Stefan-Boltzmann constant, $\beta$ is the tilt angle of the panel, $\varepsilon$ refers to the emissivity, and $h_{c, \text { forced }}$ is the forced convection coefficient. The indices for the temperatures are self-explanatory.

\section{Model Implementation}

\subsection{Electrical System}

\subsubsection{Overall System Model}

In order to model the electrical part of a PV system, the following Modelica classes were created: Current Source, Diode, PV array, and PV cell.

The PV cell class connects all of these elements and also includes the heat transfer model described below in Subsection 3.2. It includes a two-pin electrical port which is to be connected to the load and a temperature port to receive the ground temperature. Additional inputs are solar irradiance, the numerical value of the ambient temperature, and the cloud cover fraction. Parameters are propagated from its included classes. The model is shown in Figure 2 


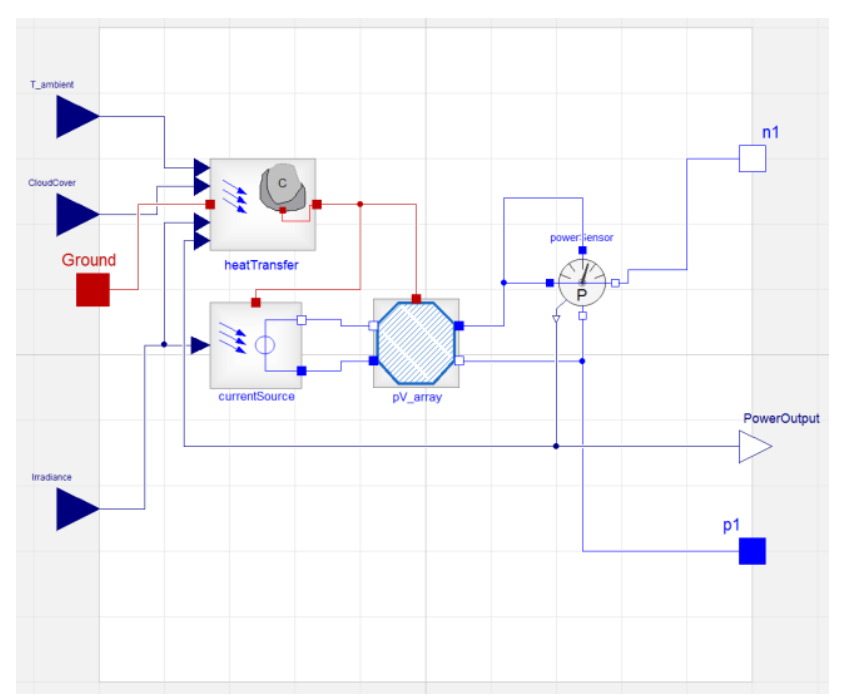

Figure 2. PV cell model.

The Current Source class has a two-pins electrical port connected to the signal-driven current source. The signal input for the source is calculated using the equation (1) using the solar irradiance as input. The class also has a thermal port that provides the temperature of the cell. Parameters for this class are the same as in the equation (1) and are propagated to the PV cell class.

$\mathrm{PV}$ array is a two-port electrical system. One port is connected to the Current Source and the other to the load. It also has a thermal port. The model includes the shunt and the series resistances and the diode model modified as described in the next Subsection. Parameters are propagated from its included classes and are further propagated to the PV cell class. The model is shown in Figure 3.

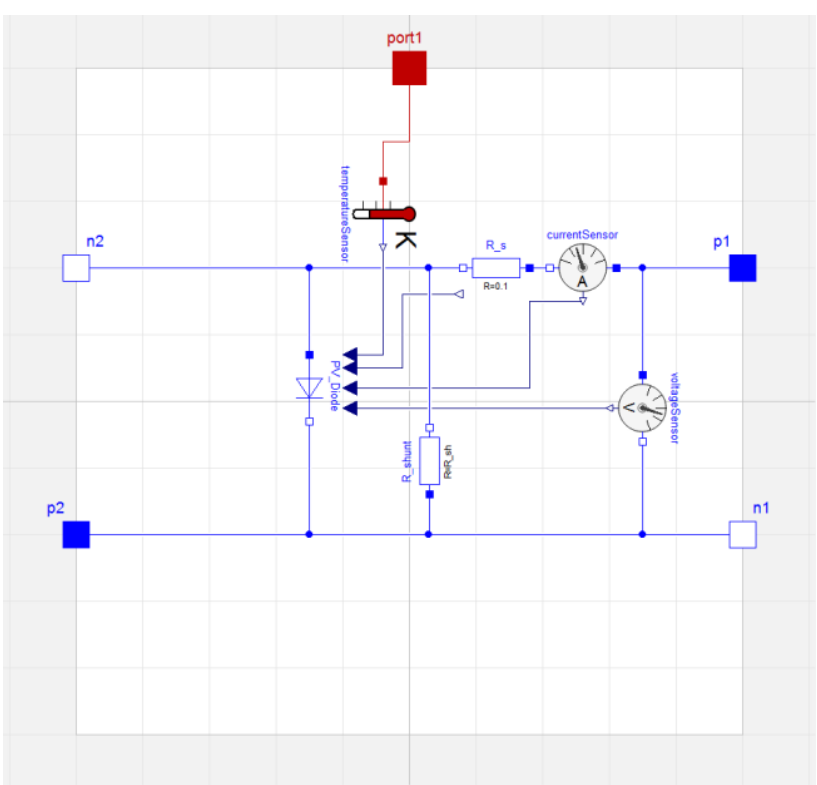

Figure 3. PV array model.

\subsubsection{Diode Model}

The PV_Diode model implements equations described in Subsection 2.3. It has been modified from the MSL Diode2 model.

The model itself has a basic electrical OnePort which are connected in the equivalent circuit model. Additionally, the model has four real inputs. These inputs are the temperature of the PV module, the serial resistance of the equivalent circuit model as well as the current and voltage signal of the load.

In comparison to the Diode2 model of the MSL we do not need to smooth the function, because we do not have to consider any switching behavior.

Parameters for the diode model and their default values are shown in Figure 4.

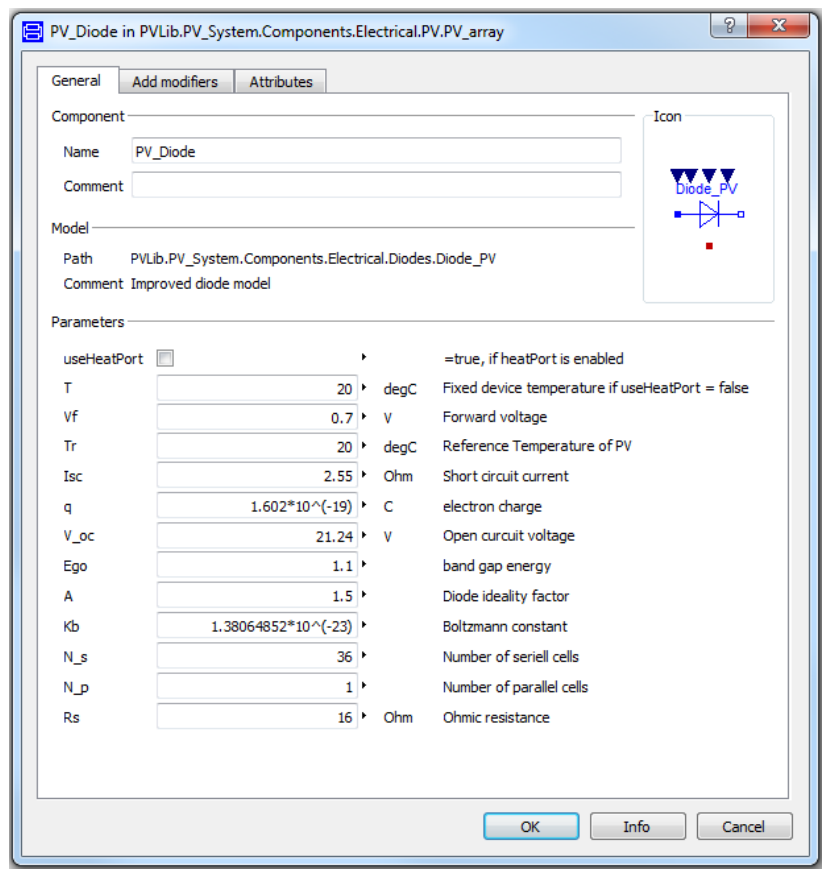

Figure 4. Parameters for the diode model.

The model equations are implemented as follows:

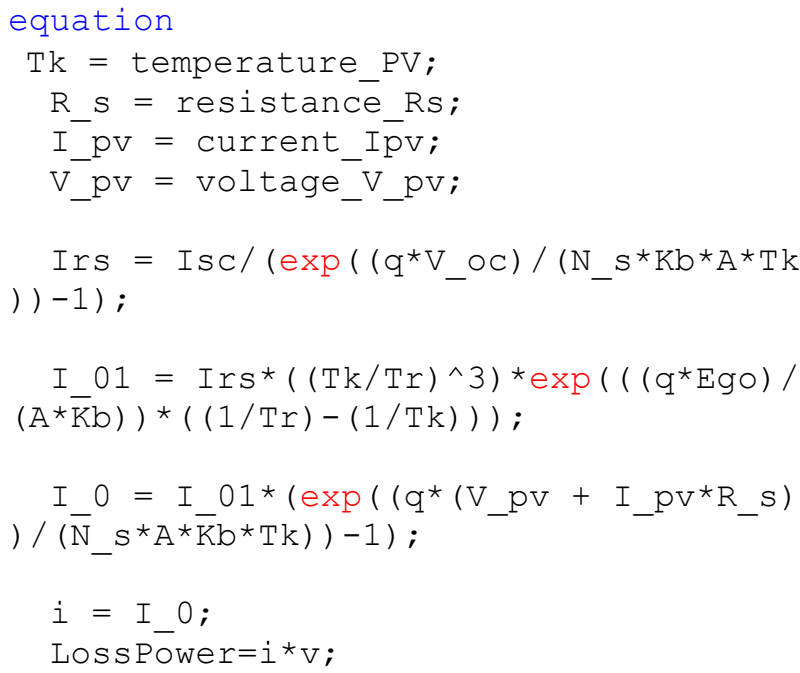




\subsubsection{Verification}

After modeling the electrical part of the PV-array, we want to verify our model. Most of the literature use the same output characteristics of PV modules to characterize the system behavior. We use these characteristics to verify the correct behavior of our model.

The tests are made at a constant temperature of $293.15 \mathrm{~K}$. Two variables are varied to get the typical curve. First the system load will be varied. Second, the irradiation will be changed.

Figure 5 shows the test setup of the verification experiment.

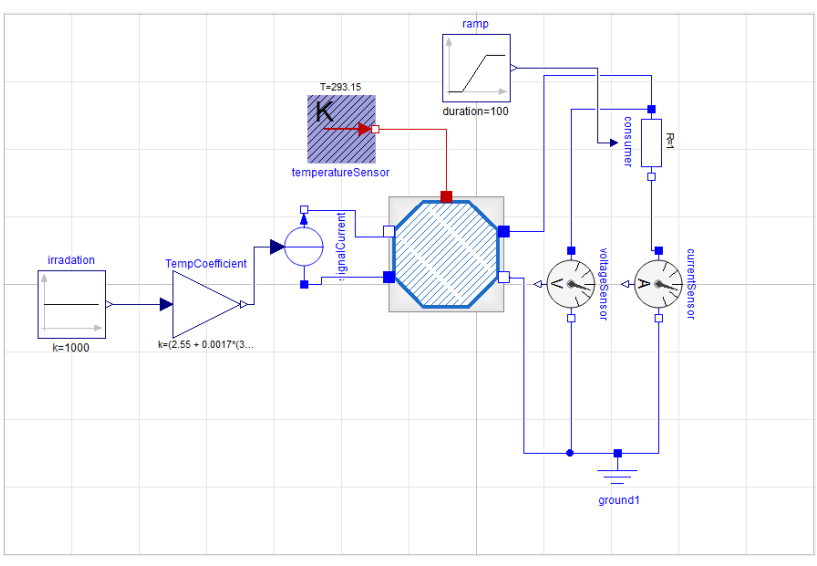

Figure 5. PV array test setup.

For the electrical parameters we use the references of a Solkar 36W PV module. The electrical characteristics can be seen in Table 1 (Pandiarajan and Muthu, 2011).

Table 1. Electrical characteristic data of Solkar 36W

\begin{tabular}{|l|r|}
\hline Electric parameters & \\
\hline Open circuit voltage $\left(\mathrm{V}_{\mathrm{OC}}\right)$ & $21.24 \mathrm{~V}$ \\
\hline Short circuit current $\left(\mathrm{I}_{\mathrm{SCr}}\right)$ & $2.55 \mathrm{~A}$ \\
\hline Number of cells in series $\left(\mathrm{N}_{\mathrm{s}}\right)$ & 36 \\
\hline Number of cells in parallel $\left(\mathrm{N}_{\mathrm{p}}\right)$ & 1 \\
\hline
\end{tabular}

For the first simulation run we take the irradiation of $200 \mathrm{~W} / \mathrm{sqm}$ and change the electrical load outside of the PV cell. This load represents the consumer. We repeat this experiment with the irradiation of $600 \mathrm{~W} / \mathrm{sqm}$ and $1000 \mathrm{~W} / \mathrm{sqm}$.

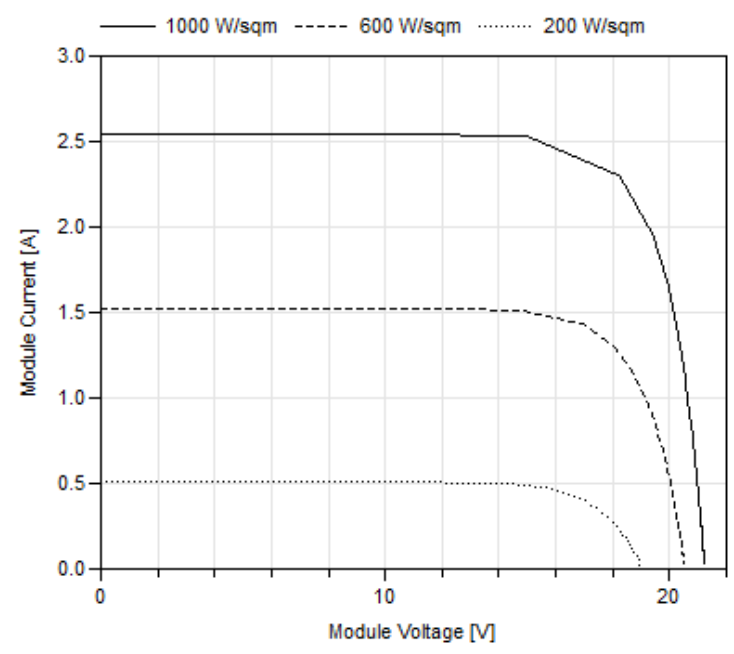

Figure 6. I-V characteristics with varying irradiation.

Figure 6 shows the result of the simulation runs. You can see the characteristic I-V output diagram of a PV cell. The results match the results from the paper (Pandiarajan and Muthu, 2011). Our electrical model is therefore considered verified.

\subsection{Thermal System}

The thermal system implements the equations described in Subsection 2.4. It has two thermal ports: one for the ground temperature and one for the temperature of the cell. The latter is connected to both Current Source and the PV array classes. Additional inputs are ambient temperature, solar irradiance, the cloud cover ratio, and the electrical power output computed in the PV cell model. Parameters for the heat transfer model are also described in Subsection 2.4 and they are propagated to the PV cell class. The model is shown in Figure 7.

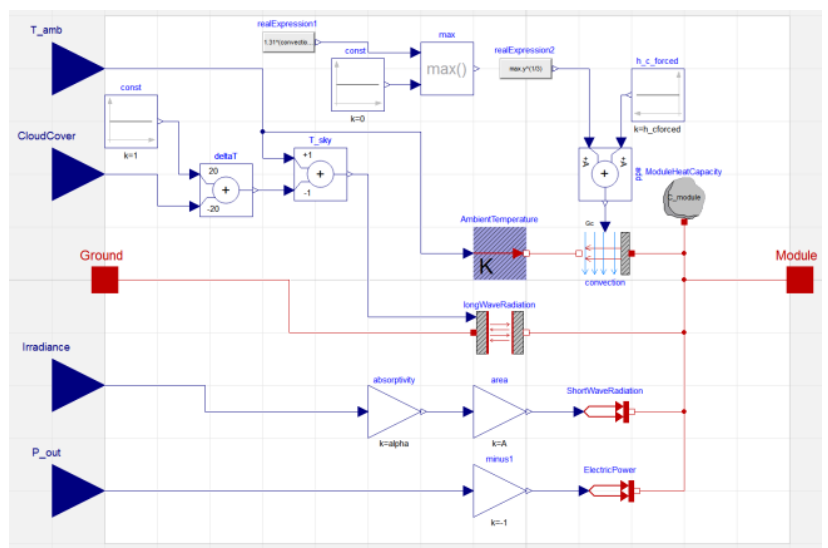

Figure 7. Heat transfer model.

Convection is modelled with the MSL Thermal Convection component. The forced convection coefficient is implemented as a parameter and the free convection coefficient is computed using the cubic root law with the provision that it can never be less than zero. 
An additional class has been created for computing the long wave radiation by modifying the MSL thermal Body Radiation component. The two thermal ports from this component are used to connect the ground and the PV cell. A real input port has been added for the temperature of the sky. The parameters for this class are emissivities, the area of the panel and the tilt angle and they are propagated to the heat transfer model. The governing equation has been modified as follows in order to comply with the equation (5):

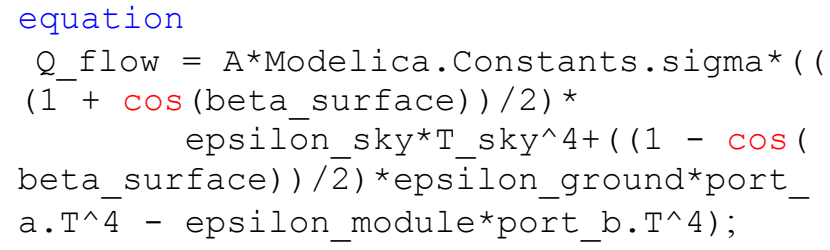

The variables in this equation correspond to those in the equation (5). For example, beta_surface means $\beta$, the tilt angle of the panel.

Unfortunately, data in (Jones and Underwood, 2001) does not provide enough information to verify our model in simulation experiments.

\section{Experiments and Results}

Simulation experiments have been run using the tool Dymola and the weather models from the HVAC Library. The setup is shown in Figure 8.

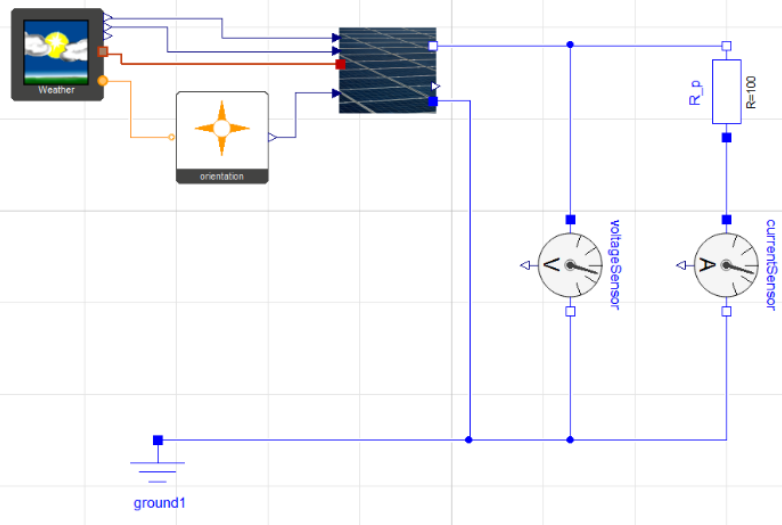

Figure 8. Experimental setup.

The weather and the orientation models are taken directly from the above-referenced library. The weather model uses the file specifying the weather condition for the entire year. It provides values of the ambient temperature, the cloud cover, the ground temperature and sun position in the sky. The latter is used by the orientation model to calculate the solar irradiance.

We have used the following values of the parameters for the PV cell as shown in Figure 9.
To get a better understanding of the system it makes sense to simulate the PV cells over the course of a year. To see the effect of the weather on the energy output of our solar system, it is interesting to compare different climate conditions. To keep the simulation results simple and comparable we only varied the cloud coverage between $0 \%$ and $50 \%$ for this investigation. The remaining weather conditions were kept the same for both simulation runs.

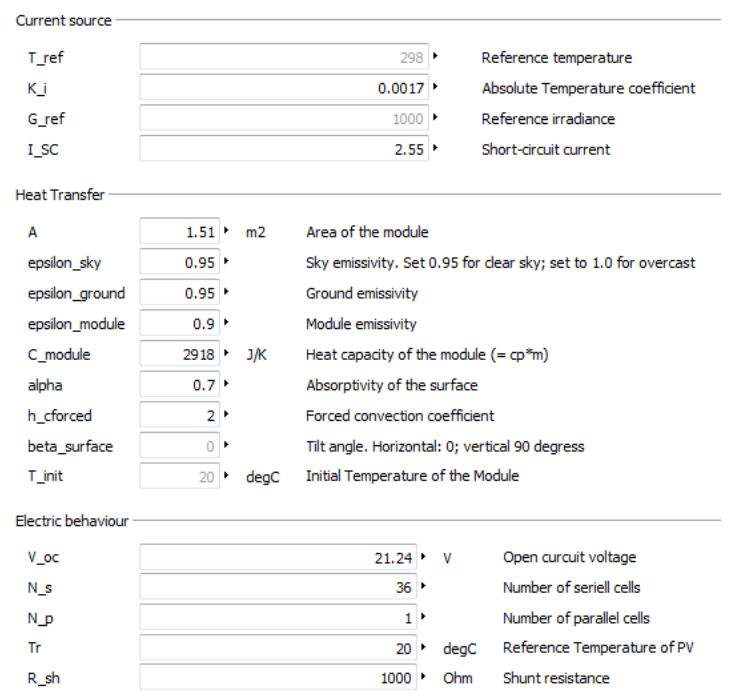

Figure 9. Parameters for the simulation.

In Figure 10 the generated energy over the course of a year is shown. One can see, that the cloud coverage has an effect on the energy output of the PV system. Although the simple variation of this weather input is just effecting the long wave radiation, described in Subsection 2.4. To get a better understanding of the electric outputs and the possible use-cases of the complete system, we would have to make simulations with varying set of weather data.

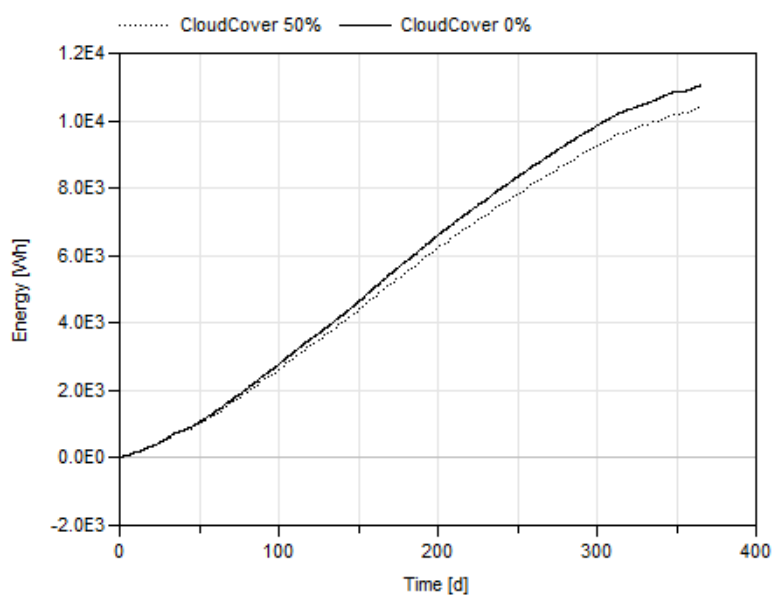

Figure 10. Electric energy over one year. 


\section{Conclusions and Outlook}

The results of the simulation experiments demonstrate the fundamental soundness of our modeling approach, which is to combine the electrical and the thermal models.

Future research may proceed along the following lines. First, it may be beneficial to consider other weather conditions and/or climates. Second, it may be of interest to implement control systems and algorithms so that various parameters can be changed in response to changing weather conditions in order to obtain maximum power output. Finally, it will be interesting to investigate the feasibility of using PV systems in electric vehicles and/or battery charging stations.

\section{References}

S. W. Angrist. Direct Energy Conversion, $4^{\text {th }}$ ed. Boston: Allyn and Bacon, Inc., 1982

L. Castaner and S. Silvestre. Modeling Photovoltaic Systems Using PSpice. Chichester: Wiley and Sons, Inc., 2002.

J. P. Holman, S. Bhattacharyya. Heat Transfer. In SI Units., $10^{\text {th }}$ ed. McGraw Hill, 2011.

A. D. Jones and C. P. Underwood. A Thermal Model for Photovoltaic Systems. Solar Energy, Vol. 70, No 4, pp. 349-359, 2001.

T. Khatib and W. Elmenreich. Modeling of Photovoltaic Systems Using MATLAB, John Wiley \& Sons, 2016.

G. M. Masters. Renewable and Efficient Electric Power Systems. Hoboken; Wiley and Sons, Inc., 2004.

N. Pandiarajan and R. Muthu. Mathematical Modeling of Photovoltaic Module with Simulink. International Conference on Electrical Energy Systems (ICEES), 3-5 Jan 2011, pp. 314-319.

M. Seyedmahmoudian, S. Mekhilef, R. Rahmani, R. Yusof and E. T. Renami. Analytical Modeling of Partially Shaded Photovoltaic Systems. Energies, 2013, 6, pp. 128-144. doi: 10.3390/en6010128.

H. Tian, F. Mancilla-David, K. Ellis, E. Muljadi and P. Jenkins. A Cell-to-Module-Array Detailed Model for Photovoltaic Panels. Solar Energy, 86, pp. 2695-2706, 2012. 